\title{
Analytical Quality-By-Design Compliant Ultrafast Liquid Chromatographic Method for Determination of Paliperidone in Extended Release Tablet Dosage Form
}

Sagar Suman Panda*, Sarwar Beg\#, Ravi Kumar, Vankata Varaha Bera and Priyadarshini Singh

Roland Institute of Pharmaceutical Sciences, Berhampur-760010, Odisha, India

\begin{abstract}
The current study deals with development and validation of a simple, fast and sensitive ultrafast liquid chromatographic method using the cardinal principles of analytical quality by design for estimation of paliperidone in extended release pharmaceutical dosage form. A Box-Behnken design was adopted for optimizing chromatographic conditions, especially the method robustness by selecting organic phase composition, mobile phase flow rate and strength of tetra butyl ammonium hydrogen sulfate solution as the factors, and evaluating their effect on the responses like, retention time and tailing factor. The chromatography was performed on a C-18 column $(250 \times 4.6$ $\mathrm{mm}, 5 \mu \mathrm{m})$ using methanol: $10 \mathrm{mM}$ tetra butyl ammonium hydrogen sulfate $(95: 5 \% \mathrm{v} / \mathrm{v})$ as mobile phase at flow rate of $1.0 \mathrm{ml} / \mathrm{min}$ with photodiode array detection at $279 \mathrm{~nm}$. Method validation studies revealed calibration curve with linearity of drug concentration ranging between 2 and $100 \mu \mathrm{g} / \mathrm{ml}$. Values of accuracy were found to be well within the acceptance limit with mean percent recovery between 98.22 and $102.96 \%$. Inter and intra-day precision showed percent RSD values were within the acceptance limits. Method sensitivity revealed that limit of detection and limit of quantitation as $0.5 \mu \mathrm{g} / \mathrm{ml}$ and $2 \mu \mathrm{g} / \mathrm{ml}$, respectively. The system suitability analysis also yielded high degree of method robustness. Overall, the UFLC method was found suitable for determination of paliperidone in bulk and pharmaceutical dosage forms.
\end{abstract}

\section{Keywords: Paliperidone; UFLC; QbD; BBD; Validation}

\section{Introduction}

Paliperidone, 3-\{2-[4-(6-fluoro-1,2-benzisoxazol-3-yl)piperidin1-yl] ethyl $\}$-9-hydroxy-2-methyl-6,7,8,9-tetrahydropyrido[1,2-a] pyrimidin-4-one (Figure 1) is a benzisoxazole derivative acts dopamine and $5-\mathrm{HT}_{2 \mathrm{~A}}$ receptor antagonist primarily and primarily exhibit antipsychotic action [1]. Several analytical methods have been developed for estimation of paliperidone in pharmaceutical dosage forms and biological samples including spectrophotometric [2-4], high performance liquid chromatography(HPLC) [5-12], ultra performance liquid chromatography (UPLC) [13-15], high performance thin layer liquid chromatography(HPTLC) $[16,17]$ and liquid chromatographymass spectrometry(LC-MS) $[18,19]$ methods. However, these reported chromatographic methods for estimation of paliperidone possess multiple drawbacks like traditional method development procedure, low sensitivity, complex mobile phase mixture, strict monitoring of critical method parameters like mobile phase flow rate, column temperature, flow gradient, maintenance of $\mathrm{pH}$, etc. This calls for development of a simple, rapid, sensitive, efficient and reliable liquid chromatographic method for quantification of paliperidone in bulk drug and pharmaceutical dosage forms. The current analytical method has several advantages over the reported chromatographic methods such as use of a simple mobile phase composition (isocratic), having

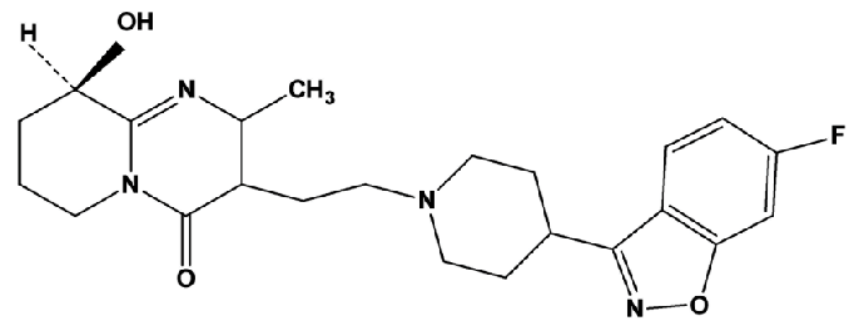

Figure 1: Chemical structure of Paliperidone. capacity to withstand various experimental stress conditions, no requirement for maintenance of $\mathrm{pH}$, wider linearity range, better sensitivity and superior robustness using principles of Quality by Design $(\mathrm{QbD})$ are a few of them.

Recently implementation of QbD paradigms for development of robust analytical methods has been highly popularized [20]. QbD is a systematic approach for the development of pharmaceutical products and processes that begins with predefined objectives and primarily emphasizes on product and process understanding based on the principles of sound science and quality risk management [21]. Since its conception and instigation by ICH and USFDA through series of guidance from Q8-Q10, QbD application for development of pharmaceutical products is mandatory [22]. Although implementation of $\mathrm{QbD}$ for analytical methods is not a federal requirement, however, the literature reports in the past a few years is a testimony to the real time benefits of $\mathrm{QbD}$ for efficient development of diverse analytical methods. The QbD approach for analytical method development involves a multistep process. It includes defining the analytical target profile (ATP) and critical analytical attributes (CAAs), identifying the

*Corresponding authors: Sagar Suman Panda, Department of Pharmaceutical Analysis and Quality Assurance, Roland Institute of Pharmaceutical Sciences, Khodasingi, Berhampur-760010, Odisha, India, Tel:+91-9438040646; E-mail: sagarguddu2002@gmail.com

"Sarwar Beg, Department of Pharmaceutics, Roland Institute of Pharmaceutical Sciences, Khodasingi, Berhampur-760010, Odisha, India, Tel: +91-9437770090; E-mail: sarwar.beg@gmail.com

Received July 08, 2015; Accepted July 31, 2015; Published August 05, 2015

Citation: Panda SS, Beg S, Kumar R, Bera VV, Singh P (2015) Analytical QualityBy-Design Compliant Ultrafast Liquid Chromatographic Method for Determination of Paliperidone in Extended Release Tablet Dosage Form. J Bioanal Biomed 7: 116-123. doi:10.4172/1948-593X.1000133

Copyright: (c) 2015 Panda SS, et al. This is an open-access article distributed under the terms of the Creative Commons Attribution License, which permits unrestricted use, distribution, and reproduction in any medium, provided the original author and source are credited. 
critical method parameters (CMPs) and their subsequent optimization using suitable experimental designs, modelization and optimum search through response surface methodology (RSM) to embark upon the analytical design space, and postulation of control strategy for continuous improvement [23].

To implement QbD in analytical method development, at first the analyte of interest and the intended purpose of the method should be defined clearly. It includes identification of the target, sample condition and setting up the quantification criteria. The second phase includes selection of a suitable analytical technique based on the need of the method intent and setting up the method performance criteria. The third phase involves assessment of possible risk by quality risk management (QRM) approach to find out the critical method parameters and possible variations in the sample integrity. A well designed risk assessment provides necessary information on the method variables which further needs investigation or a suitable control strategy [24]. The fourth phase consists of developing of a robust and efficient analytical method using a suitable Design of Experiments (DoE). The DoE is widely used for performing statistically well designed experiments to evaluate the method superiority and for getting quality results. It provides knowledge about the design space to work within for achieving quality. The design space is an experimental safe zone where the method variables have no significant influence on the quality of product. Response surface methods like Factorial Design (FD), Fractional Factorial Design (FFD), Central Composite Design (CCD) and Box-Behnken Design (BBD) are used for predicting and optimizing the responses for most of the analytical techniques [25-28]. These designs help the analytical scientists to understand the relationship between different method variables and their effect on the responses produced. The BBD has been used in analytical sciences for optimization of extraction, derivatization and separation for chromatographic methods [29-31]. BBD has several advantages than compared with other statistical design approaches such as ability to optimize $3^{3}$-Factorial designs, needs fewer numbers of total experiments, rotatibility and possess less physical constraints for experimentation [32]. Therefore, it can be effectively applied for navigating the design space for developing a robust UFLC method.

The fifth phase of QbD involves setting up control strategies to meet the desired method performance by defining the control space and system suitability. The final phase involves a continuous surveillance to gain more knowledge on the design space and updating the process with most recent innovations to further improve the method quality $[33,34]$.

The objective of present research work, therefore, was to overcome the drawbacks of reported methods for developing a UFLC method for quantification of paliperidone in bulk drug and extended release tablet dosage form using the $\mathrm{QbD}$ approach. The $\mathrm{BBD}$ was applied for systematic development and optimization of analytical method to ensure robustness. The validation studies [35] were performed for linearity, accuracy, precision, specificity, limit of detection, limit of quantitation and to derive system suitability test (SST) limits [36]. Further, the developed method was applied for estimation of paliperidone in extended release tablet dosage forms.

\section{Materials and Methods}

\section{Materials and reagents}

Methanol (Merck Ltd., Mumbai, India) was of HPLC grade. Tetrabutyl ammonium hydrogen sulfate (TBAHS) was procured from
Hi-Media Pvt. Ltd., Mumbai. The water for HPLC was obtained by using TKA-GENPURE Water Purifier, Germany. Paliperidone (purity $>$ 98.5\%) was obtained as gift sample from Ranbaxy Laboratories Ltd., India. Purity of the standard drug was assessed by testing the melting point. Further, extended release tablet dosage form (Aspire XR) containing $6 \mathrm{mg}$ of paliperidone was analyzed by UFLC.

\section{Chromatographic conditions}

A binary gradient UFLC with two SHIMADZU PROMINENCE UFLC LC-20AD pumps, a $20 \mu \mathrm{l}$ sample injection loop (manual) and SPD M20A photo diode array (PDA) detector was used. Signal was recorded and integrated using LC Solution Software. Chromatographic separation was performed on an Enable C-18G, $(250 \times 4.6 \mathrm{~mm}, 5 \mu \mathrm{m})$ column at room temperature. The mobile phase was methanol: $10 \mathrm{mM}$ TBAHS solution $(95: 5 \% \mathrm{v} / \mathrm{v})$ at a flow rate of $1.0 \mathrm{ml} / \mathrm{min}$ in isocratic mode. The $10 \mathrm{mM}$ TBAHS solution was prepared by accurately weighing $1.70 \mathrm{~g}$ of salt and dissolving it in $200 \mathrm{ml}$ of HPLC grade water. Finally the volume was made up to $500 \mathrm{ml}$ with HPLC grade water. TBAHS solution was filtered through a $0.45 \mu \mathrm{m}$ filter to remove particulate matter, if any. Afterwards, both the methanol and TBAHS solution were ultra-sonicated up to 20 minutes for degassing before use. PDA detection was carried out at $279 \mathrm{~nm}$. The injection volume was $20 \mu \mathrm{l}$. The run time was 10 minutes for all the solutions. The BBD was generated, and test data was analyzed using Design-Expert 8.0.6 Software Full Version with Serial No. 1019-4550(Stat-Ease, Inc., 2021 East Hennepin Ave., Suite 480, Minneapolis, MN 55413, USA).

\section{Establishment of analytical target profile}

A thorough survey of available literatures and drug profile was carried out for establishment of analytical target profile (ATP) containing a dynamic summary of quality characteristics of an analytical method. This primarily encompassed development of a fast, sensitive, reliable and efficient analytical method for estimation of selected drug candidate paliperidone in pharmaceutical dosage forms. Therefore, based on the prime objectives of this study, an UFLC method was adopted. The rationale justification for selection of UFLC method was framed as rapid analysis of drug as compared to conventional HPLC methods.

\section{Preparation of calibration curve}

A standard stock solution of paliperidone was prepared by transferring $25 \mathrm{mg}$ of drug in to $25 \mathrm{ml}$ volumetric flask having $10 \mathrm{ml}$ of mobile phase and was ultrasonicated for 5 minutes. Finally the volume was made up with methanol, which gave $1000 \mu \mathrm{g} / \mathrm{ml}$ solution. For preparation of calibration curve, suitable aliquots of the stock solution were taken at concentrations 2 to $100 \mu \mathrm{g} / \mathrm{ml}$ for paliperidone and finally diluted with mobile phase. A sample volume of $20 \mu \mathrm{l}$ was injected in triplicate, from each standard solution and chromatograms were recorded. Calibration curve was prepared selecting the concentration $(\mu \mathrm{g} / \mathrm{ml})$ on $\mathrm{x}$-axis and average peak areas on y-axis. The calibration curve data was further subjected to regression analysis to find out the regression statistics.

\section{Preparation of sample solution}

Tablet powder equivalent to $25 \mathrm{mg}$ of paliperidone was transferred into a $25 \mathrm{ml}$ volumetric flask, containing $10 \mathrm{ml}$ of mobile phase and ultrasonicated for 45 minutes to facilitate complete dissolution of the drug and its subsequent extraction into the solvent. The volume was made up and mixed well. Solution was filtered by $0.45 \mu \mathrm{m}$ filter to remove particulate matter, if any. The filtered solution was further 
properly diluted for analysis, to get a test concentration of $20 \mu \mathrm{g} / \mathrm{ml}$. All the solutions were stored at $2-8^{\circ} \mathrm{C}$ temperature up to 2 days for future use.

\section{Establishment of cause-effect relationship}

Ishikawa fish-bone diagram being one of the simplest tools, helpful in understanding the cause-and-effect relationship among the potential method variables, which influences the method performance. In this regard, the fish-bone diagram was drawn by highlighting diverse method variables plausibly influencing the method attributes of UFLC method of paliperidone. In the present studies, a Cause-Effect Risk Assessment Matrix with Control-Noise-Experimentation (CNX) approach was utilized for identifying the high risk variable affecting the analytical attributes. Three critical method variables viz. percentage methanol, mobile phase flow rate and strength of TBAHS solution were identified with highest scores and then subjected to response surface optimization to create a design space for developing a robust and reliable UFLC method. From this, vital method attributes affecting the method performance were shortlisted and subjected for further analysis employing suitable experimental designs.

\section{DoE guided method development and data analysis}

Based on the prior knowledge and risk assessment, three critical method variables were selected owing to their significant influence on the method performance. Methanol proportion (A), mobile phase flow rate (B) and strength of TBAHS solution (C) were considered as the independent method variables, which needed further study to test the method robustness. Fifteen experimental runs with minimal three centers points were obtained as per the BBD. Experiments were performed on UFLC in a randomized order to get bias free response. A standard concentration $10 \mu \mathrm{g} / \mathrm{ml}$ was used for all the experimental runs. The obtained experimental runs were evaluated for retention time $\left(\mathrm{Y}_{1}\right)$ and tailing factor $\left(\mathrm{Y}_{2}\right)$ as the response variables. The obtained data for the experimental runs was fitted to a suitable mathematical model by multiple linear regression analysis (MLRA) using DesignExpert Software. The developed model was allowed to study both the main effects and interaction effects. The obtained data for each experimental run was fitted to a suitable mathematical model as per the MLRA. The developed model was allowed to study both the main effects and interaction effects. Only the coefficients of model terms found to be significant $(p<0.05)$ as per analysis of variance (ANOVA) analyses were considered in framing the polynomial equation followed by analyzing the model for parameters like lack of fit, coefficient of correlation $\left(\mathrm{R}^{2}\right)$ and predicted error sum of squares (PRESS) for apt model fitting. Response surface analysis was carried out through 2D-contour plots and 3D-response surface plots for understanding the relationship among factor(s) and response(s). The search for optimum chromatographic solution was embarked upon by numerical optimization desirability function in the studied range.

\section{Developing method control strategy}

Defining the method performance through system suitability tests provide the working limits for critical method variables, which may affect the system suitability adversely. In this study SST limits for retention time, number of theoretical plates and tailing factor were derived to develop the method control strategy. The SST limits can be efficiently derived from the experimental results of robustness study. For a replicated experiment, the system suitability limits can be described as the upper or lower limit from one-sided $95 \%$ confidence interval around a response mean. For parameters like retention time, the lower limit was selected, while for tailing factor it would be the upper limit. The equation - 1 and equation- 2 can be used to determine the confidence interval for the lower limit and the upper limit, respectively.

$$
\begin{aligned}
& \{\{\bar{Z}-t \alpha, n-1 \cdot(s / \sqrt{n})\} \\
& \{\{\bar{Z}+t \alpha, n-1 \cdot(s / \sqrt{n})\}
\end{aligned}
$$

Where $\bar{Z}$, average of three observations $t_{\alpha, n-1}=t_{\text {critical }(\alpha=0.05, n-1=\operatorname{deg} \text { rees of freedom }), \mathrm{S} \text {, standard deviation of }}$ three observations and, $\sqrt{n}$, square root of number of observations.

\section{Method validation}

The developed method was validated as per the $\mathrm{ICH}$ guidance for specificity, linearity, accuracy, precision, solution stability, Limit of Detection (LOD) and Limit of Quantitation (LOQ). Specificity of the method was determined by checking interference due to addition of commonly used excipients by comparing the chromatograms of blank, placebo, standard paliperidone and paliperidone in formulation (Figure 2). To find out accuracy of the method, recovery studies were carried out at $80 \%, 100 \%$ and $120 \%$ of the test concentration $(20 \mu \mathrm{g} /$ $\mathrm{ml}$ ) of paliperidone by standard addition method. The recovery study was performed thrice at each level. To assess the intraday, interday and system precision, six replicates of a fixed concentration $(20 \mu \mathrm{g} /$ $\mathrm{ml}$ ) drug solution were injected onto the column and percent RSD values were calculated. Stability of drug solution $(20 \mu \mathrm{g} / \mathrm{ml})$ stored under refrigerated environment was determined by the developed chromatographic method, by injecting onto the column in triplicate after every $24 \mathrm{~h}$. The obtained results were compared with those obtained from freshly prepared standard solutions. The LOD and LOQ were determined based on visual detection of Signal to Noise ratio. For limit of detection, the $\mathrm{S} / \mathrm{N}$ ratio was taken as 3:1. For limit of quantitation, the $\mathrm{S} / \mathrm{N}$ ratio was taken as 10:1.

\section{Results and Discussion}

The early UFLC method development needs pertinent information on the prior knowledge and experience about the chromatographic parameters with physical and chemical properties of the analyte. A C-18 column was used for separation of the compound as it was found suitable based on chemical properties of analyte. Methanol and $10 \mathrm{mM}$ TBAHS solution $(95: 5 \% \mathrm{v} / \mathrm{v})$ when employed as mobile phase with a flow rate of $1.0 \mathrm{ml} / \mathrm{min}$ produced a symmetrical peak for paliperidone at retention time of $2.64 \mathrm{~min}$. Further, to study the different factors affecting separation of paliperidone and to get quality for method reliability and robustness, QbD approach was implemented.

Evaluation of associated risk with the help of Ishikawa fishbone diagram (not shown in figure) revealed identification of the primary causes and secondary sub-causes, which are responsible for the variability and alteration in method performance. This could be attributed because of the variation in analyst, instruments, environment, methodology, materials and measurement. The Ishikawa diagram also addresses selecting critical method variables which can affect the method performance adversely and helps in carrying out an effective risk assessment. In the present studies, a Cause-Effect Risk Assessment Matrix with CNX approach was utilized (Table 1). The critical method variables (CMVs) were identified based on the final score followed by response surface optimization of them to create the design space for developing a robust and reliable UFLC method. 

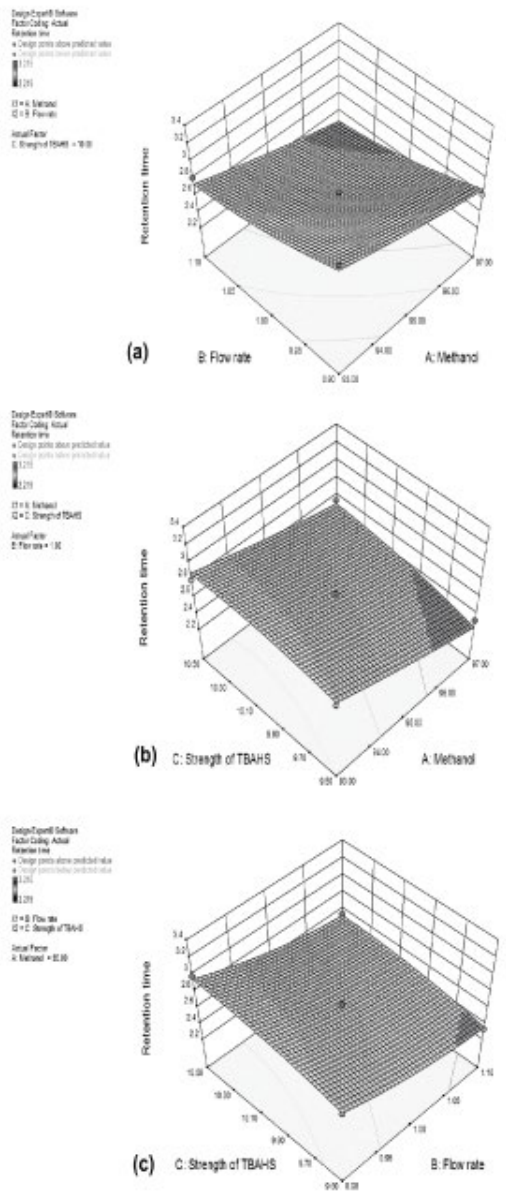
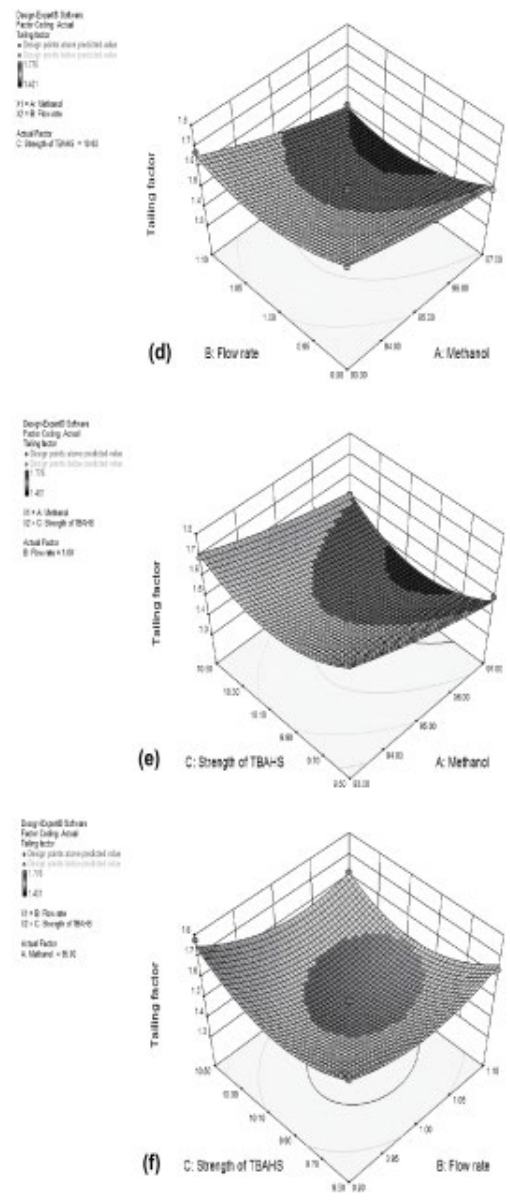

Figure 2: Typical overlaid chromatogram of (a) blank, (b) placebo, (c) standard paliperidone and (d) paliperidone in extended release tablet dosage form.

Based on the prior knowledge and risk assessment studies, three critical method variables were decided to have significant influence on method performance. Organic phase composition (A), mobile phase flow rate (B) and strength of TBAHS solution (C) were the three variables, which needed further study to assess the method robustness. Fifteen experimental runs obtained as per BBD model were performed on a random basis using UFLC and responses were obtained to assure method robustness for identifying optimized method conditions. Table 2 shows the responses obtained from the experimentation and the levels of chromatographic variables A and B were studied.

A second-order model with a quadratic experimental domain was adopted. Evaluation of the model showed highly statistical significant difference between the model terms $(p<0.05)$, insignificant lack of fit, high $\mathrm{R}^{2}$ value and lower values of PRESS. The ANOVA results for the experimental model revealed highly a significant difference in the values achieved by all the three responses. Further, the polynomial regression equations generated for each of the studied responses $\mathrm{Y}_{1}$ and $\mathrm{Y}_{2}$ were assessed for the model terms and interacting variables. Coefficients for each model terms were analyzed to identify influence of each variable and their interactions on magnitude of responses. Model equations for the studied response variables are as given below:

Retention time $\left(Y_{1}\right)=+2.63-0.24 A-0.21 B+0.092 C+0.003 A B+$ $0.047 A C-0.00625 B C+0.019 A^{2}+0.063 B^{2}-0.048 C^{2}$
Tailing factor $\left(Y_{2}\right)=+1.46-0.11 A-0.039 B-0.002 C-0.012 A B+$ $0.019 A C-0.025 B C+0.019 A^{2}+0.088 B^{2}+0.13 C^{2}$

where, $\mathrm{A}$ is methanol (\%), $\mathrm{B}$ is mobile phase flow rate $(\mathrm{ml} / \mathrm{min})$ and $\mathrm{C}$ is strength of TBAHS solution (mM)

A positive sign of the coefficient suggests a synergistic effect, whereas a negative sign indicates antagonistic effect. Evaluation of equation- 3 and 4 suggests that the coefficient of each model terms were statistically significant $(p<0.05)$ indicating apt fitting of the obtained mathematical data to the design.

The results of ANOVA for response $Y_{1}$ revealed that the predicted values for the three factors are within the satisfactory value with the predicted $F$-value of 14.44, which implies the model is significant. Model $p$-value suggests that there is only a $0.45 \%$ chance that model $F$-value can be large due to noise. In case of response $\mathrm{Y}_{2}$, the ANOVA results indicates the predicted values for all the factors with a predicted model $F$-value of 12.35 suggesting that the model is significant and only a $0.64 \%$ chance of obtaining high model $F$-value because of noise. The least squares regression values were highest for the responses $Y_{1}$ $\left(R^{2}=0.9629\right)$ and $\mathrm{Y}_{2}\left(R^{2}=0.9569\right)$ than compared to the other models. The adequate precision is a measure of signal to noise ratio. Ratio values higher than 4.0 suggests model aptness to navigate through the design space (Table $\mathrm{S} 1$ ).

Response surface analysis was carried out employing 3D-response 
Citation: Panda SS, Beg S, Kumar R, Bera VV, Singh P (2015) Analytical Quality-By-Design Compliant Ultrafast Liquid Chromatographic Method for Determination of Paliperidone in Extended Release Tablet Dosage Form. J Bioanal Biomed 7: 116-123. doi:10.4172/1948-593X.1000133

surface plot and 2D-contour plots for identifying the underlying interaction(s) among the studied factors. The 3D-response surface plot illustrated in Figure 3a for retention time shows a linear declining value with increase in the methanol (\%) at high levels of flow rate. Similarly, in case of Figure $3 b$, a linear declining value with increase in methanol (\%) at low levels of strength of TBAHS was noticed. Figure $3 \mathrm{c}$ represents a declining trend with increase in flow rate at low levels of strength of TBAHS. Figure 3d shows a gradual declining trend is noticed in the tailing factor with increasing levels of methanol (\%) from low to high levels at mid-levels of flow rate. However, there was no effect on tailing factor due to change in flow rate at different levels.
Figure $3 \mathrm{e}$ depicted a decrease in tailing factor at high levels of methanol (\%) and mid-levels of strength of TBAHS solution. A higher tailing factor was observed at high levels of strength of TBAHS and low levels of methanol (\%). Figure $3 \mathrm{f}$ indicated a considerable decrease in tailing factor with a typical "saddle point" at around mid-levels of flow rate and strength of TBAHS solution (Figure 3).

The similar interpretation was also revealed from the corresponding 2D-contour plots (not shown in figure). The contour plot for retention time suggested decrease in retention time with increase in the levels of methanol and flow rate. The ideal retention time value was observed

\begin{tabular}{|c|c|c|c|c|c|c|}
\hline \multirow[t]{2}{*}{ Method Parameter } & \multirow[t]{2}{*}{ Cause } & \multicolumn{2}{|c|}{ Effect of Risk Level on CMAs } & \multirow[t]{2}{*}{ Total Score* } & \multirow[t]{2}{*}{$\mathrm{C}, \mathrm{N}, \mathrm{X}^{\mathrm{a}}$} & \multirow[t]{2}{*}{ Strategy } \\
\hline & & Retention time & Tailing factor & & & \\
\hline Mobile Phase & Methanol \% & 10 & 10 & 200 & $x$ & DoE \\
\hline Pump & Flow rate & 10 & 10 & 200 & $x$ & DoE \\
\hline Mobile phase & Strength of TBAHS & 10 & 8 & 180 & $x$ & DoE \\
\hline Column & Phase & 4 & 4 & 80 & $\mathrm{C}$ & C-18 \\
\hline Column & Age & 4 & 4 & 80 & $\mathrm{~N}$ & New Column \\
\hline Mobile Phase & Solvent grade & 4 & 3 & 70 & C & HPLC grade \\
\hline Column & Equilibration & 2 & 3 & 50 & $\mathrm{C}$ & Controlled \\
\hline
\end{tabular}

Table 1: C-E based risk assessment.

${ }^{\mathrm{a}} \mathrm{C}, \mathrm{N}, \mathrm{X}$-Controlled, Noise, Experimental ${ }^{\star}$ Total Score $=($ Risk level of First CMA $\times 10)+($ Risk level of Second CMA $\times 10)$ where, Score: 1-Negligible risk, 5-Low risk, 10-High risk

\begin{tabular}{|c|c|c|c|}
\hline Run No. & Methanol (\%): A & Flow rate $(\mathrm{ml} / \mathrm{min})$ : B & Strength of TBAHS (mM): C \\
\hline 1 & 0 & 0 & 0 \\
\hline 2 & -1 & -1 & 0 \\
\hline 3 & 0 & 0 & 0 \\
\hline 4 & 0 & -1 & +1 \\
\hline 5 & +1 & -1 & 0 \\
\hline 6 & -1 & 0 & -1 \\
\hline 7 & 0 & +1 & -1 \\
\hline 8 & 0 & +1 & +1 \\
\hline 9 & 0 & 0 & 0 \\
\hline 10 & +1 & +1 & 0 \\
\hline 11 & +1 & 0 & +1 \\
\hline 12 & 0 & -1 & -1 \\
\hline 13 & -1 & 0 & +1 \\
\hline 14 & -1 & +1 & 0 \\
\hline 15 & +1 & 0 & -1 \\
\hline Coded Level & -1 (Low) & 0 (Intermediate) & +1 (High) \\
\hline Variable A: & 93 & 95 & 97 \\
\hline Variable B: & 0.9 & 1.0 & 1.1 \\
\hline Variable C: & 9.5 & 10.0 & 10.5 \\
\hline
\end{tabular}

Table 2: Experimental design matrix for robustness study.

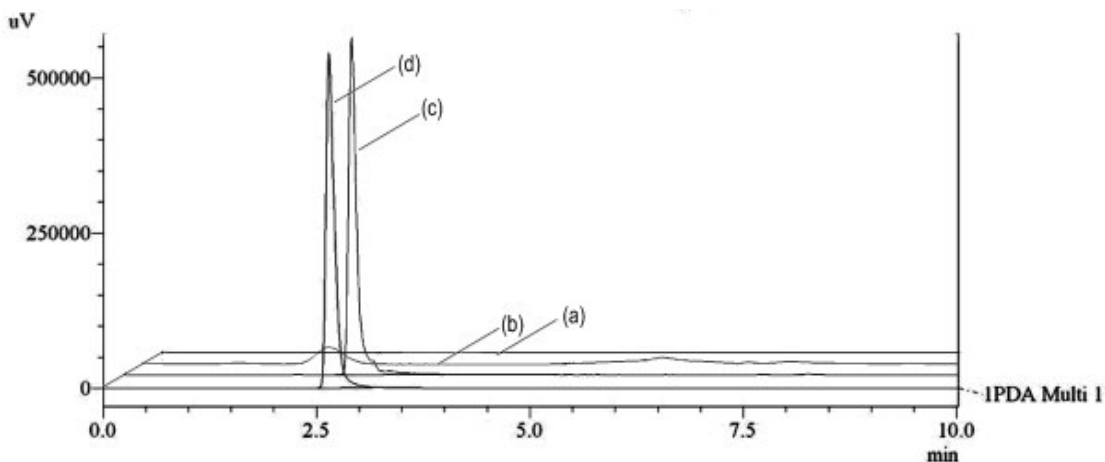

Figure 3: 3D Response surface plots for Retention time (Y1) and Tailing factor (Y2) against methanol percentage vs. flow rate - (a), (d); methanol percentage vs. strength of TBAHS-(b),(e); flow rate vs. strength of TBAHS - (c),(f). 


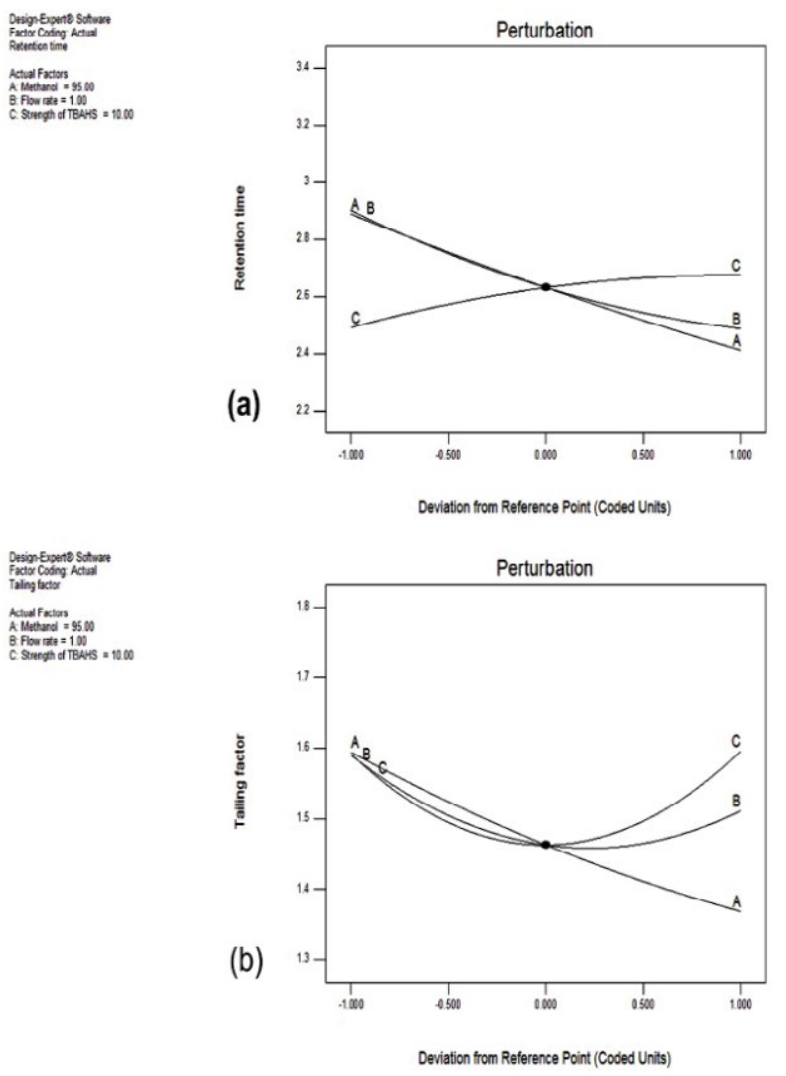

Figure 4: Perturbation graphs showing effects of variables on responses (a) Retention time- $Y_{1}$, (b) Tailing factor- $Y_{2}[A=$ Methanol $(\%) ; B=F l o w$ rate $(\mathrm{mL} / \mathrm{min})$ and $\mathrm{C}=$ Strength of TBAHS $(\mathrm{mM})]$.

at the intermediate levels of both the factors. A decrease in retention time was noticed with increasing levels of methanol and low levels of strength of TBAHS, whereas, the retention time decreased gradually with increase in flow rate at low levels of strength of TBAHS. In case of tailing factor, the contours depicted a gradual declining trend with high levels of methanol at mid-levels of strength of TBAHS solution. Tailing was found to decrease at high levels of methanol (\%) and mid-levels of strength of TBAHS. However, there was no prominent change in tailing at different levels of flow rate and strength of TBAHS solution. A minima was also noticed at mid-levels of both the factors. Like response surfaces, all the contour plots also showed the presence of curved contour lines, thus revealed the prevalence of interactions among the factors on the studied response variables.

Perturbation plots show the effect of an independent variable on a specific response, while holding other variables constant at the reference point. In all the plots (Figure 4), a direct intersection of lines for each factor confirmed presence of interaction. A curvature or steep slope for each line corresponding to a factor indicated response sensitiveness towards a particular variable. Upon in-depth analysis of the trend observed in perturbation plots, a synergistic relationship was observed with negative influence among all the three factors with sharp and linearly declining trend. The perturbation plot for theoretical plate, the factor A (methanol, \%) and factor C (strength of TBAHS) presented a curvilinear trend with presence of interaction between each other. However, the factor B (flow rate) revealed a negative influence on the obtained response. Unlike the trend observed for retention time, all the factors indicated positive influence on the response tailing factor with prevalence of interaction effect among the studied factors.

Further, the experimental data was evaluated with the help of model diagnostic plots to check the appropriate fitting of data and for identifying the abnormalities, if any. Overall, various model diagnostic plots like, predicted vs. actual plot, residual vs. run plot, Cook's distance plot and Box-Cox plot (not shown in figure) revealed excellent fit of experimental data to the selected experimental design and mathematical model with absence of outliers.

The developed model was subjected to optimization module in Design Expert software for determining the optimized values for all three factors A, B and C leading to maximal or minimal values for the responses indicating optimal method performance. The optimized solutions obtained from mathematical optimization employing desirability function (Desirability value $=1$ ) suggested to proceed the method at the nominal values $(A=95 \%$ methanol, $B=$ flow rate at 1.0 $\mathrm{ml} / \mathrm{min}$ and $\mathrm{C}=$ TBAHS strength at $10 \mathrm{mM}$ ).

The developed method was validated for specificity, linearity, accuracy, precision, LOD, LOQ and system suitability. Specificity of the method was assured by comparing the obtained chromatograms of blank, placebo, standard paliperidone and paliperidone in extended release tablet dosage form. Figure 4 indicates non-interference of any of the commonly used excipients in the estimation process. The calibration curve was found to be linear over a concentration range of $2-100 \mu \mathrm{g} / \mathrm{ml}$ for with linear regression equation $\mathrm{y}=32685 \mathrm{x}+92933$. Regression analysis of linearity data showed overall goodness-offit. The values obtained for statistical parameters such as Multiple $R$, $R^{2}$, Adjusted $R^{2}$ and standard error were 0.9994, 0.9989, 0.9988 and 41715.51 , respectively. ANOVA suggested appropriateness ( $p$-value < 0.05 ) of the linearity data. The values for recovery studies show high levels of accuracy of the method (Table S2). The method was found to be precise as the RSD (\%) values for intraday, interday and system precision were below $2 \%$, confirming method preciseness (Table S3). The result of solution stability showed no significant degradation or change in paliperidone content indicating, stability of solutions up to 2 days under refrigeration. The average $(\mathrm{n}=3)$ recoveries were $100.21 \%$ $(\mathrm{RSD}=0.49 \%)$ and $97.65 \%(\mathrm{RSD}=0.23 \%)$, for day 1 and 2 , respectively. The LOD and LOQ values were found to be $0.5 \mu \mathrm{g} / \mathrm{ml}$ and $2 \mu \mathrm{g} / \mathrm{ml}$, respectively. Further, derivation of system suitability limits (Table 3 ) helped defining the method control strategies. Statistical interpretation of experimental design data and results of SST limits suggested a better robustness within the controlled method operable range for the most influential method variables such as, methanol proportion $( \pm 2 \%)$, flow rate $( \pm 0.1 \mathrm{ml} / \mathrm{min})$ and strength of TBAHS solution $( \pm 0.5 \mathrm{mM})$.The result of assay $(n=3)$ from the tablet dosage form for paliperidone yielded $100.1 \%(\mathrm{SD}= \pm 1.4)$ of drug. The higher percentage of recovery and noninterference of the formulation excipients in retention time of the drug shows the selectivity of the method for estimation of paliperidone in extended release tablet dosage form.

\begin{tabular}{|c|c|c|}
\hline Run & Retention Time (min) & Tailing Factor \\
\hline $\mathbf{1}$ & 2.642 & 1.467 \\
\hline $\mathbf{2}$ & 2.633 & 1.461 \\
\hline $\mathbf{3}$ & 2.635 & 1.498 \\
\hline Mean & 2.636 & 1.510 \\
\hline S.D. & 0.0038 & 0.0162 \\
\hline $\mathbf{( n )}$ & 3 & 3 \\
\hline SST-Limits & 2.636-2.92 $(0.0038 / \sqrt{ } 3)=2.629$ & $1.510+2.92(0.0162 / \sqrt{ } 3)=1.537$ \\
\hline & Table 3: Derivation of system suitability limits. \\
\hline
\end{tabular}




\section{Conclusion}

The present research paper explains a novel approach for quantification of paliperidone in pharmaceutical dosage form by imposing the principles of $\mathrm{QbD}$. Implementation of $\mathrm{QbD}$ approach ensured that quality is built into the analytical method, ensuring robustness and reliability of the method. Use of a BBD for robustness study revealed that a slight change in method parameters can affect the responses. This suggests the analyst to take suitable precautionary control measures to minimize the unwanted effect of these noise variables. Further this method may be continuously monitored to further improvise any of the method performance criteria. Results for validation parameters were within the acceptance limits. Therefore, the method is suitable for routine analysis of paliperidone in bulk drug and pharmaceutical dosage form. Further this method may be applied for estimation of paliperidone in biological fluids.

\section{Acknowledgement}

The authors are thankful to Ranbaxy Laboratories Pvt. Ltd. for providing the reference standard of Paliperidone and Roland Institute of Pharmaceutical Sciences, Khodasingi, Odisha, India for providing the necessary research facilities.

\section{References}

1. Sweetman SC (2009) Martindale- The Complete Drug Reference. (36 $6^{\text {th }}$ edition) Pharmaceutical Press, London.

2. Trivedi RK, Jupudi R, Patel MC, Trivedi DR, Jogia HA (2013) A validated spectrophotometric method for determination of paliperidone palmitate in bulk drug and its pharmaceutical dosage form. Res Rev J Pharm Pharma Sciences 2: $37-41$.

3. Korrapati U, Chintala R (2014) Spectrophotometric methods for the quantitative estimation of paliperidone in formulations. Chem Sci Trans 3: 1156-1162.

4. Injavarapu D, Panigrahy UP (2014) New method development and validation of UV-spectrophotometer for the estimation of paliperidone in bulk and pharmaceutical dosage form. Int J Pharm Res BioSci 3: 106-125.

5. Jadav SA, Landge SB, Choudhari PM, Solanki PV, Bembalkar SR, et al (2011) Stress degradation behavior of paliperidone, an antipsychotic drug, and development of suitable stability-indicating RP-LC method. Chromatogr Res Int 2011: 1-10.

6. Dadare K, Khatri NC, Pant Mayank (2012) Application of RP-RRLC methods for estimation of paliperidone in tablet dosage forms. J Chem Pharm Res 4 3154-3157.

7. Manjula devi AS, Ravi TK (2012) A validated RP-HPLC method for the estimation of paliperidone in bulk and tablet dosage form. Am J Pharm Tech Res 2: 616-626.

8. Farooqui M, Ahmed RZ, Sangshetti JN, Zaheer Z, Wahab A, et al. (2013) Identification and determination of related substances of paliperidone in bulk drug and pharmaceutical formulations by HPLC and HPLC-MS-MS. Indo Am J Pharm Res 3: 3175-1383

9. Kancharla S, Pavan GS, Paladugu ND, Bonthu S, Poloju D, et al. (2013) Development and validation of paliperidone in bulk and pharmaceutical formulation by RP-HPLC. Int J Biol Pharm Res 4: 412-416.

10. Umamahesswar K, Ramu G, Rambabu C (2013) A reverse phase HPLC method development and validation for the determination of paliperidone in pure and dosage forms. Chem Sci Trans 2: 41-46.

11. Rao KN, Ganapathy S, Rao AL (2013) Development and validation of new HPLC method for the estimation of paliperidone in pharmaceutical dosage forms. Rasayan J Chem 6:34-38.

12. Boga $H$, Patnaik A (2014) A novel method for chromatographic determination of paliperidone in pure form and formulation by using RP-HPLC and its stability studies. Int J Innov Pharm Sci Res 2: 2709-2718.

13. Bindu KH, Reddy IU, Anjaneyulu Y, Suryanarayana MV (2012) A stabilityindicating ultra-performance liquid chromatographic method for estimation of related substances and degradants in paliperidone active pharmaceutical ingredient and its pharmaceutical dosage forms. J Chrom Sci 50: 368-372.
14. Bindu KH, Dhekale NH, Suryanarayana MV, Anjaneyulu Y (2012) A validated stability indicating UPLC method for simultaneous determination of assay, related substances, and degradation products of paliperidone palmitate active pharmaceutical ingredient and its pharmaceutical injection forms. J Chromatogr Relat Technol 35: 533-546.

15. Trivedi RK, Jain P, Patel MC, Chatrabhuji PM, Trivedi DR (2013)A rapid, stability indicating RP-UPLC method for determination of paliperidone palmitate in depot injectable formulation. J Appl Pharm Sci 3: 087-092.

16. Patel RB, Patel MR, Bhatt KK, Patel BG (2010) HPTLC method development and validation: $Q$ uantification of paliperidone in formulations and in vitro release study. Anal Method 2: 525-531.

17. Pawar SM, Dhaneswar SR (2012) Validated high performance thin laye chromatographic method for quantitation of paliperidone in the presence of degradation products formed under ich recommended stress conditions. J Pharm Biomed Sci 16: 1-5.

18. Bocato MZ, Simões RA, Calixto LA, de Gaitani CM, Pupo MT, et al. (2012) Solid phase micro extraction and LC-MS/MS for the determination of paliperidone after stereo selective fungal biotransformation of risperidone. Anal Chim Acta 742: 80-89.

19. Chen $\mathrm{H}$, Zhao L, Li G, Leng $\mathrm{D}$, Ma $\mathrm{P}$, et al. (2014) Development and validation of a rapid and sensitive UHPLC-MS/MS method for the determination of paliperidone in beagle dog plasma. Asian J Pharm Sci 9: 286-292.

20. Monks KE, Rieger HJ, Molnár I (2011) Expanding the term "Design Space" in high performance liquid chromatography (I). J Pharm Biomed Anal 56: 874-879.

21. International Conference on Harmonization (ICH) (2009) Pharmaceutica Development Q8. (R2) IFPMA, Geneva

22. Rathore AS, Bhambure R, Ghare V (2010) Process analytical technology (PAT) for biopharmaceutical products. Anal Bioanal Chem 398: 137-154.

23. Lionberger RA, Lee SL, Lee L, Raw A, Yu LX (2008) Quality by design Concepts for ANDAs. AAPS J 10: 268-276.

24. International Conference on Harmonization (ICH) (2005) Quality Risk Management Q9. IFPMA, Geneva.

25. Krier F, Brion M, Debrus B, Lebrun P, Driesen A, et al. (2011) Optimization and validation of a fast HPLC method for the quantification of sulindac and its related impurities. J Pharm Biomed Anal 54: 694-700.

26. Bianchini RM, Castellano PM, Kaufman TS (2009) Development and validation of an HPLC method for the determination of process-related impurities in pridinol mesylate, employing experimental designs. Anal Chim Acta 654: 141-147.

27. Deshpande GR, Roy AK, Rao NS, Rao BM, Reddy JR (2011) Rapid screening of volatile ion-pair reagents using UHPLC and robust analytical method development using DoE for an acetyl cholinesterase inhibitor: Galantamine $\mathrm{HBr}$. Chromatographia 73: 639-648.

28. Ihssane B, Charrouf M, Aborriche A, Abboud Y, Bouabidi A, et al. (2011) Optimization and validation of a new high-performance liquid chromatographic method for analysis of acetazolamide in tablets using box-behnken statistical experiment design. Acta Chromatogr 23: 41-57.

29. Gfrerer M, Lankmayr E (2005) Screening, optimization and validation of microwave-assisted extraction for the determination of persistent organochlorine pesticides. Anal Chim Acta 533: 203-211.

30. Stafiej A, Pyrzynska K, Ranz A, Lankmayr E (2006) Screening and optimization of derivatization in heating block for the determination of aliphatic aldehydes by HPLC. J Biochem Biophys Methods 69: 15-24.

31. Servais AC, Fillet M, Chiap P, Abushoffa AM, Hubert P, et al. (2002) Optimization of the separation of $ß$-blockers by ion-pair capillary electrophoresis in nonaqueous media using univariate and multivariate approaches. J Sep Sci 25 1087-1095

32. Mason RL, Gunst RF, Hess JL (2003) Statistical Design and Analysis of Experiments: With Applications to Engineering and Science. ( $2^{\text {nd }}$ edition) John Wiley and Sons Inc, Hoboken, New Jersey.

33. International Conference on Harmonization (ICH) (2008) Pharmaceutical Development Q10. IFPMA, Geneva.

34. International Conference on Harmonization (ICH) (2012) Development and Manufacture of Drug Substances Q11. IFPMA, Geneva. 
Citation: Panda SS, Beg S, Kumar R, Bera VV, Singh P (2015) Analytical Quality-By-Design Compliant Ultrafast Liquid Chromatographic Method for Determination of Paliperidone in Extended Release Tablet Dosage Form. J Bioanal Biomed 7: 116-123. doi:10.4172/1948-593X.1000133

35. International Conference on Harmonization (ICH) (2005) Validation of Analytical Procedures: Text and Methodology Q2 (R1). IFPMA, Geneva
36. Heyden YV, Jimidar M, Hund E, Niemeijer N, Peeters R, et al. (1999) Determination of system suitability limits with a robustness test. J Chromatogr A 845: 145-154. 